\title{
Front Matter: Volume 10679
}

, "Front Matter: Volume 10679," Proc. SPIE 10679, Optics, Photonics, and Digital Technologies for Imaging Applications V, 1067901 (13 August 2018); doi: $10.1117 / 12.2503293$

SPIE. Event: SPIE Photonics Europe, 2018, Strasbourg, France 


\section{PROCEEDINGS OF SPIE}

\section{Optics, Photonics, and Digital Technologies for Imaging Applications $V$}

\section{Peter Schelkens Touradj Ebrahimi Gabriel Cristóbal Editors}

\section{4-26 April 2018 \\ Strasbourg, France}

Sponsored by

SPIE

Cosponsored by

Strasbourg the Europtimist (France)

CNRS (France)

Investissements d'Avenvir (France)

iCube (France)

Université de Strasbourg (France)

Cooperating Organisations

Photonics 21 (Germany)

EOS_European Optical Society (Germany)

Photonics Public Private Partnership (Belgium)

Comité National d'Optique et de Photonique (France)

Published by

SPIE 
The papers in this volume were part of the technical conference cited on the cover and title page. Papers were selected and subject to review by the editors and conference program committee. Some conference presentations may not be available for publication. Additional papers and presentation recordings may be available online in the SPIE Digital Library at SPIEDigitalLibrary.org.

The papers reflect the work and thoughts of the authors and are published herein as submitted. The publisher is not responsible for the validity of the information or for any outcomes resulting from reliance thereon.

Please use the following format to cite material from these proceedings:

Author(s), "Title of Paper," in Optics, Photonics, and Digital Technologies for Imaging Applications $V$, edited by Peter Schelkens, Touradj Ebrahimi, Gabriel Cristóbal, Proceedings of SPIE Vol. 10679 (SPIE, Bellingham, WA, 2018) Seven-digit Article CID Number.

ISSN: 0277-786X

ISSN: 1996-756X (electronic)

ISBN: 9781510618848

ISBN: 9781510618855 (electronic)

Published by

SPIE

P.O. Box 10, Bellingham, Washington 98227-0010 USA

Telephone +1 3606763290 (Pacific Time) · Fax +1 3606471445

SPIE.org

Copyright (c) 2018, Society of Photo-Optical Instrumentation Engineers.

Copying of material in this book for internal or personal use, or for the internal or personal use of specific clients, beyond the fair use provisions granted by the U.S. Copyright Law is authorized by SPIE subject to payment of copying fees. The Transactional Reporting Service base fee for this volume is $\$ 18.00$ per article (or portion thereof), which should be paid directly to the Copyright Clearance Center (CCC), 222 Rosewood Drive, Danvers, MA 01923. Payment may also be made electronically through CCC Online at copyright.com. Other copying for republication, resale, advertising or promotion, or any form of systematic or multiple reproduction of any material in this book is prohibited except with permission in writing from the publisher. The CCC fee code is $0277-$ $786 \mathrm{X} / 18 / \$ 18.00$.

Printed in the United States of America.

Publication of record for individual papers is online in the SPIE Digital Library.

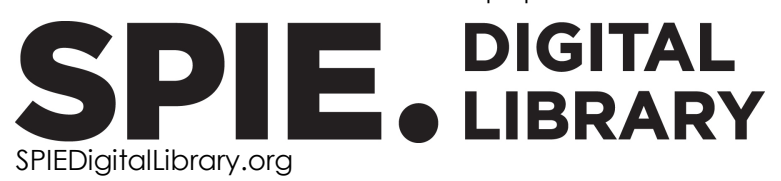

Paper Numbering: Proceedings of SPIE follow an e-First publication model. A unique citation identifier (CID) number is assigned to each article at the time of publication. Utilization of CIDs allows articles to be fully citable as soon as they are published online, and connects the same identifier to all online and print versions of the publication. SPIE uses a seven-digit CID article numbering system structured as follows:

- The first five digits correspond to the SPIE volume number.

- The last two digits indicate publication order within the volume using a Base 36 numbering system employing both numerals and letters. These two-number sets start with 00, 01, 02, 03, 04, 05, 06, 07, 08, 09, OA, OB ... 0Z, followed by 10-1Z, 20-2Z, etc. The CID Number appears on each page of the manuscript. 


\title{
Contents
}

\author{
ix Authors \\ xiii Conference Committee
}

\section{SESSION 1 MEDICAL IMAGING I}

1067902 Deep features using convolutional neural network for early stage cancer detection [10679-4]

1067903 Quality enhancement of multispectral images for skin cancer optical diagnostics [10679-1]

1067904 Monitoring of the excretion of fluorescent nanocomposites out of the body using artificial neural networks [10679-2]

1067905 Cloud infrastructure for skin cancer scalable detection system [10679-3]

1067906 Probing tissue multifractality for optical diagnosis of diabetic retinopathy [10679-5]

\section{SESSION 2 MEDICAL IMAGING II}

1067907 Visual measurement of any shape of the flaccid membrane of the extracorporeal pneumatic heart assist pump [10679-7]

10679 OA Classification of brain tissue with optical coherence tomography by employing texture analysis [10679-6]

SESSION $3 \quad$ MICROSCOPIC IMAGING

10679 OC The generation of optical needle and magnetization needle with tunable longitudinal depth [10679-16]

10679 OD Fluorescence imaging for whole slide scanning using LED-based color sequential illumination [10679-11]

10679 OE Axial scanning and spherical aberration correction in confocal microscopy employing an adaptive lens [10679-12]

10679 OG Lights and piffalls of convolutional neural networks for diatom identification [10679-14] 
$10679 \mathrm{OH} \quad$ Image scanning microscopy (ISM) with a single photon avalanche diode (SPAD) array detector [10679-15]

SESSION $4 \quad$ PLENOPTIC IMAGE PROCESSING

$10679 \mathrm{Ol} \quad$ Advanced mobile three-dimensional display based on computer-generated integral imaging [10679-21]

10679 0J Information processing challenges of full parallax light field displays [10679-17]

10679 OK Benchmarking coding standards for digital holography represented on the object plane [10679-18]

10679 OL View-dependent compression of digital holograms based on matching pursuit (Best Student Paper) [10679-20]

10679 OM Compression scheme for sparsely sampled light field data based on pseudo multi-view sequences [10679-19]

10679 ON Rapid vertical tissue imaging with clinical multiphoton tomography [10679-81]

SESSION $5 \quad$ DISPLAY SYSTEMS

1067900 Optical flow-based filtering for effective presentation of the enhanced vision on a HUD [10679-24]

10679 OP Laser speckle reduction based on partial spatial coherence and microlens-array screens [10679-22]

$106790 Q \quad$ Camera-based color measurement of DLP projectors using a semi-synchronized projector camera system [10679-23]

10679 OR Conception of a touchless human machine interaction system for operating rooms using deep learning [10679-80]

SESSION 6 HOLOGRAPHIC IMAGING AND RENDERING

10679 OS Image blur and visual perception for rainbow holographic display [10679-26]

10679 OT Diffraction elements by nanoparticle composites for holographic display [10679-29]

10679 OU Spatial frequency response of nanocomposite holographic gratings [10679-27]

10679 OV Holographic stereogram printer for computer-generated holograms [10679-28]

iv 
10679 OW Curved detectors for wide field imaging systems: impact on tolerance analysis [10679-30]

10679 OX Real-time spatially adaptive deconvolution for CFA images [10679-31]

$10679 \mathrm{OZ}$ Real-time high-speed motion blur compensation method using galvanometer mirror for shape sensing of microfabricated objects [10679-33]

1067910 Curved CMOS sensor: characterization of the first fully functional prototype [10679-34]

$1067911 \quad$ Optical feedback interferometry applied to visualize an acoustic stationary wave [10679-35]

\section{SESSION 8 QUALITY ASSESSMENT}

1067912 The device for automated quality inspection and authentication of security holograms [10679-40]

1067913 How to measure image quality of virtual reality cameras in practice [10679-36]

1067915 Automating the surface inspection on small customer-specific optical elements [10679-38]

1067916 3D measurement of yarn hairiness via multi-perspective images [10679-39]

\section{SESSION 9 IMAGE ANALYSIS}

1067918 Face recognition method for cases of an insufficient training set, using 3D models of face that were created using two facial images [10679-41]

1067919 Fast object recognition method from random measurements of compressive sensing camera [10679-44]

10679 1A Research on method of vision navigation for mobile robot in unstructured environment [10679-42]

\section{POSTER SESSION}

10679 1C Hough transform-based image processing algorithm in the optical-electronic angle measuring device [10679-47] 
10679 1D Influence of noise on the estimation method to reject damped sinusoidal vibrations in adaptive optics systems [10679-48]

10679 1E Augmented logarithmic Gaussian process regression methodology for chlorophyll prediction [10679-49]

10679 IF Block reconstruction of object image based on compressed sensing and orthogonal modulation [10679-50]

10679 1G Optical design and unification of objectives: CCF PlanApo for $28 \mathrm{~mm}$ observation in the microscope [10679-51]

$106791 \mathrm{H} \quad$ A multiphase active contour model based on the Hermite transform for texture segmentation [10679-52]

$106791 \mathrm{~K} \quad$ Particle image models for optical flow-based velocity field estimation in image velocimetry [10679-55]

$10679 \mathrm{lL} \quad$ Fluid flow measurements using optical flow velocity field estimation and LED-based light sheet illumination [10679-56]

$106791 \mathrm{M} \quad$ Freeform optics complexity estimation: comparison of methods [10679-57]

10679 iN Endogenous two- and three-photon fluorescence of a biological substance with a picosecond ultrawide band laser source [10679-58]

10679 IP The optimal planning methods of remote sensing experiment in IR-range for the satellite meteorology problems [10679-60]

$106791 Q \quad$ Sparse matrix-based image enhancement for target detection using deep learning [10679-61]

10679 IR A method for automatic analysis of scanned 3D models of human hands and feet [10679-62]

10679 is Multi-sensor array for polarimetric light-field imaging [10679-63]

$106791 \mathrm{E} \quad$ Electrochromic tunable filters based on nanotubes with viologen incorporation [10679-66]

$106791 \mathrm{~V}$ Opto-electronic scanner for operational control of security holograms authenticity on documents [10679-67]

10679 IW Analysis of light-guide parameters for multicolor augmented reality indicator based on DOEs [10679-68]

$106791 Y \quad$ Asymmetric optical encryption technique implementing spatially incoherent illumination [10679-70]

1067912 Digital hologram quality improvement by elimination of imaging sensor noise [10679-71]

1067920 Deep learning object recognition in multi-spectral UAV imagery [10679-72] 
$1067921 \quad$ Methods of entering of compensation distortions into images for projection on nonplanar surfaces by development of systems of augmented reality [10679-73]

1067924 Pattern recognition based on analysis of the summary ellipse polarization state in the Fraunhofer diffraction area [10679-76]

1067927 Dense disparity estimation based on the bi-dimensional empirical mode decomposition and Riesz transformation [10679-78] 
Proc. of SPIE Vol. 10679 1067901-8

Downloaded From: https://www.spiedigitallibrary.org/conference-proceedings-of-spie on 26 Apr 2023 Terms of Use: https://www.spiedigitallibrary.org/terms-of-use 


\section{Authors}

Numbers in the index correspond to the last two digits of the seven-digit citation identifier (CID) article numbering system used in Proceedings of SPIE. The first five digits reflect the volume number. Base 36 numbering is employed for the last two digits and indicates the order of articles within the volume. Numbers start with 00, 01, 02, 03, 04, 05, 06, 07, 08, 09, 0A, 0B...0Z, followed by 10-1Z, 20-2Z, etc.

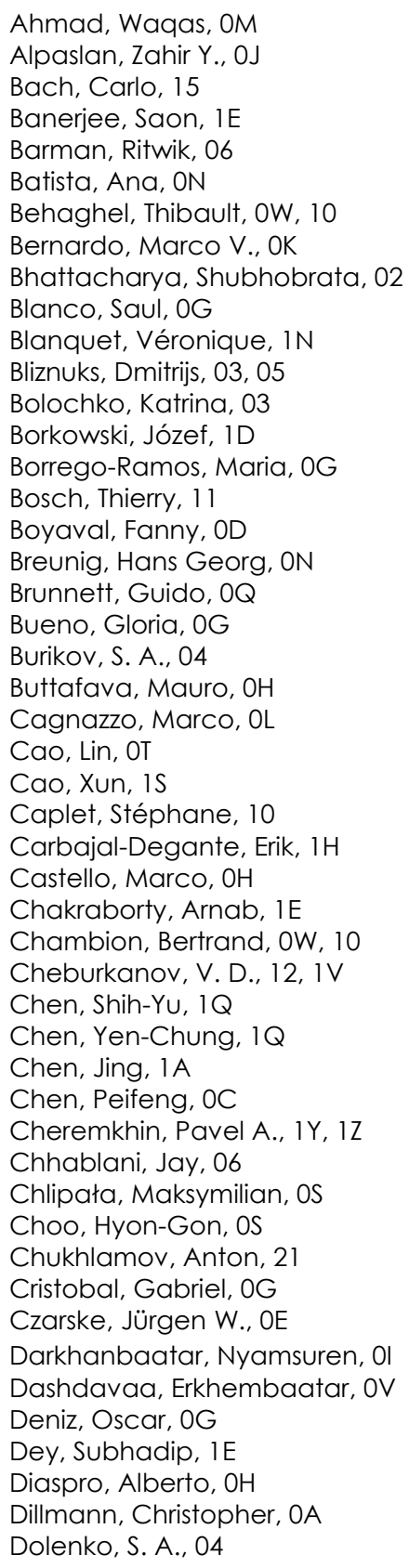

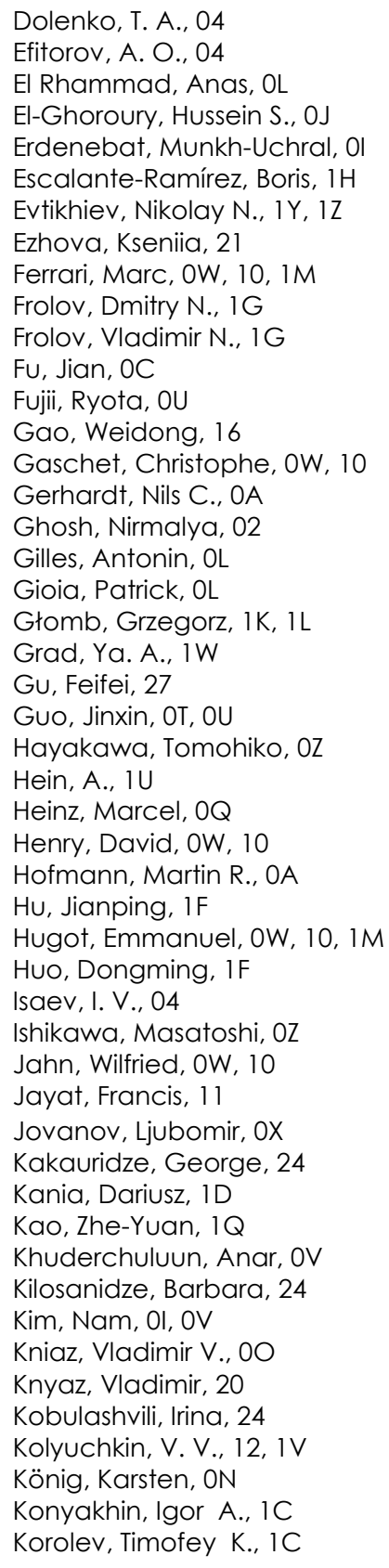


Kortz, C., $1 \mathrm{U}$

Koukourakis, Nektarios, OE

Kovalovs, M., 1R

Kowerko, Danny, OQ

Kozacki, Tomasz, OS

Krasnov, Vitaly $V_{\text {., }} 1 \mathrm{Y}, 1 \mathrm{Z}$

Krug, Robin, OA

Krutikova, O., 18

Kwon, Ki-Chul, Ol

Laptinskiy, K. A., 04

Lefort, Claire, $1 \mathrm{~N}$

Lemke, Florian, $\mathrm{OE}$

Lenz, Marcel, OA

Leon, Jerome Pitogo de, $0 Z$

Lévêque, Philippe, $1 \mathrm{~N}$

Li, Chuanrong, 19

Li, Jinxi, IF

Li, Yunqian, IS

Lihachov, Alexey, 03, 05

Lihacova, Ilze, 03

Lim, Young-Tae, OV

Linz-Dittrich, Sabine, 15

Lombardo, Simona, OW, 10, $1 \mathrm{M}$

Ma, Lingling, 19

Magnol, Laetitia, 1N

Minaeva, Ekaterina D., IY

Mukherjee, C. K., IE

Mukherjee, Sukanya, 06

Mukhopadhyay, Sabyasachi, 02, 06

Murakami, Kenichi, $\mathrm{OZ}$

Murawski, Krzysztof, 07

Muslimov, Eduard, OW, 10, 1M

Naiden, L. A, IV

Nakamura, Toshihiro, OU

Nie, Yunsong, $1 \mathrm{~S}$

Nikolaev, V. V., IW

Nogin, Anton A., 1C

Odinokov, S. B., 12, 1V, $1 \mathrm{~W}$

Oesterschulze, E., $1 \mathrm{U}$

Olenici, Adriana, OG

Olsson, Roger, OM

Olveres, Jimena, $1 \mathrm{H}$

Osipovs, Pavels, 05

Panigrahi, Prasanta K., 02, 06

Pasupuleti, Gautham, 02, 06

Pauwels, Jaël, OP

Pedraza, Anibal, OG

Peltoketo, Veli-Tapani, 13

Perchoux, Julien, 11

Pereira, Manuela, OK

Pereme, Florian, OR

Perez, Patric, 15

Pesquet-Popescu, Béatrice, OL

Philipp, Katrin, OE

Philips, Wilfried, OX

Piao, Yan-Ling, $\mathrm{Ol}$

Pinheiro, António M. G., OK

Piryutin, N. V., IV

Prabhakar, N., 04

Pratiher, Sawon, 02, 06, $1 \mathrm{E}$
Radoux, Jean-Pierre, OR

Rizky, Agyl F., OU

Rodin, Vladislav G., 1Y, 12

Rong, LU, OT

Rosenholm, J. M., 04

Roulet, Melanie, OW, 10, $1 \mathrm{M}$

Ruiz-Santaquiteria, Jesus, OG

Sanchez, Carlos, OG

Sarmanova, O. E., 04

Saver, Benjamin, ON

Scavazzin, Mirko, OR

Schelkens, Peter, OJ

Schmieder, Kirsten, OA

Schöch, Alexander, 15

Sen Karaman, D., 04

Sheppard, Colin J. R., $\mathrm{OH}$

Shifrina, Anna V., IY

Shin, Chang-Won, OV

Sisojevs, A., 18

Sjöström, Mårten, $O M$

Sokolov, Anton A., IP

Solomashenko, A. B., IW

Song, Zhan, 27

Stallinga, Sjoerd, OD

Starikov, Rostislav S., $1 Z$

Sulej, Wojciech, 07

Świrniak, Grzegorz, 1K, $1 \mathrm{~L}$

Talalaev, V. E., 12, 1V

Tang, Lingli, 19

Tomita, Yasuo, OU

Tortarolo, Giorgio, $\mathrm{OH}$

Tosi, Alberto, $\mathrm{OH}$

Tronche, Clement, 11

Tsyganov, I. K., 12, $1 \mathrm{~V}$

Urgiles, Fernando, 11

Uvarov, Nikolay V., IP

Vakulov, Pavel S., IG

Valentini, Franck, OR

van der Graaff, Leon, OD

van Vliet, Lucas J., OD

Verschaffelt, Guy, OP

Vicidomini, Giuseppe, $\mathrm{OH}$

Villa, Federica, $\mathrm{OH}$

Vinogradova, Olga A., $1 G$

Wallrabe, Ulrike, $\mathrm{OE}$

Wang, Dayong, OT

Wang, Lei, 16

Wang, Qi, 19

Wang, Ying, 0C

Wapler, Matthias C., OE

Welp, Hubert, OA

Xiao, Hong, 1 A

$X u, B u g a o, 16$

$\mathrm{XU}$, Xiaodong, $1 \mathrm{~A}$

Yanev, A. S., IW

Yang, Fu-Ming, 1Q

Ye, Chuan, $1 \mathrm{~A}$

Yuan, Sheng, $1 \mathrm{~F}$

Yue, Tao, $1 \mathrm{~s}$

Zegarra Flores, Jesus, OR 
Zhang, Luozhi, $1 \mathrm{~F}$

Zhang, Xinping, OT

Zhang, Yi, IA

Zhao, Hong, 27

Zhao, Juan, 27

Zhao, Liming, 1A

Zheltov, Sergey, 20

Zhou, Xin, $1 \mathrm{~F}$

Zhou, Yongsheng, 19

Zhou, Yuanyuan, $1 \mathrm{~F}$

Zi, Chongde, 1S

Ziolek, Carsten, 15

Zlokazov, E. Y., 12, $1 \mathrm{~V}$

Proc. of SPIE Vol. 10679 1067901-11

Downloaded From: https://www.spiedigitallibrary.org/conference-proceedings-of-spie on 26 Apr 2023 Terms of Use: https://www.spiedigitallibrary.org/terms-of-use 
Proc. of SPIE Vol. 10679 1067901-12 Downloaded From: https://www.spiedigitallibrary.org/conference-proceedings-of-spie on 26 Apr 2023
Terms of Use: https://www.spiedigitallibrary.org/terms-of-use 


\title{
Conference Committee
}

\author{
Symposium Chairs
}

Francis Berghmans, Vrije Universiteit Brussel (Belgium)

Thierry Georges, Oxxius SA (France)

Harald Giessen, Universität Stuttgart (Germany)

Paul C. Montgomery, Université de Strasbourg (France)

Conference Chairs

Peter Schelkens, Vrije Universiteit Brussel (Belgium)

Touradj Ebrahimi, Ecole Polytechnique Fédérale de Lausanne (Switzerland)

Gabriel Cristóbal, Consejo Superior de Investigaciones Científicas (Spain)

\section{Conference Programme Committee}

Olivier Aubreton, Université de Bourgogne (France)

Jan T. Bosiers, Teledyne DALSA (Netherlands)

Daping Chu, University of Cambridge (United Kingdom)

Jana Dittmann, Otto-von-Guericke-Universität Magdeburg (Germany)

Marek Domanski, University of Poznan (Poland)

Boris Escalante-Ramírez, University Nacional Autónoma de México (Mexico)

Pascuala García-Martínez, Universidade de València (Spain)

Laurent Jacques, Universiteit Catholique de Louvain (Belgium)

Tom R. L. Kimpe, Barco N.V. (Belgium)

Tomasz Kozacki, Warsaw University of Technology (Poland)

Dragan Kukolj, RT-RK Institute for Computer Based Systems (Serbia)

Jukka-Tapani Mäkinen, VTT Technical Research Centre of Finland (Finland)

Maria S. Millán García-Varela, Universidade Politècnica de Catalunya (Spain)

Stuart W. Perry, Canon Information Systems Research (Australia)

Pasi Saarikko, Oculus VR, LLC (United States)

Martin Schrader, Nokia Research Centre (Finland)

Lea Skorin-Kapov, University of Zagreb (Croatia)

Colin James Richard Sheppard, National University of Singapore (Singapore)

Athanassios N. Skodras, University of Patras (Greece) 
Andrew G. Tescher, AGT Associates (United States)

Frédéric Truchetet, Université de Bourgogne (France)

Gerald Zauner, FH OÖ Forschungs \& Entwicklungs GmbH (Austria)

\section{Session Chairs}

1 Medical Imaging I

Boris Escalante-Ramírez, Universidade Nacional Autónoma de México (Mexico)

2 Medical Imaging II

Boris Escalante-Ramírez, Universidade Nacional Autónoma de México (Mexico)

3 Microscopic Imaging

Gloria Bueno, Universidade de Castilla-La Mancha (Spain)

$4 \quad$ Plenoptic Image Processing

Peter Schelkens, Vrije Universiteit Brussel (Belgium)

5 Display Systems

Jukka-Tapani Mäkinen, VTT Technical Research Centre of Finland Ltd. (Finland)

6 Holographic Imaging and Rendering

Jukka-Tapani Mäkinen, VTT Technical Research Centre of Finland Ltd. (Finland)

7 Image Acquisition

Olivier Aubreton, Université de Bourgogne (France)

8 Quality Assessment

Peter Schelkens, Vrije Universiteit Brussel (Belgium)

9 Image Analysis

Peter Schelkens, Vrije Universiteit Brussel (Belgium) 